\title{
Electron Microscope Analyses of Self-aligned HgTe Nanocrystallites Induced by Controlled Precipitation Technique
}

\author{
Man-Jong Lee \\ Agency for Defense Development, Yousung P.O. Box 35, Daejeon 305-600, Korea \\ E-mail : manjonglee@add.re.kr
}

(Received 19 June 2002, Accepted 1 August 2002)

\begin{abstract}
Controlled precipitation of quasi-binary semiconductor system is newly proposed as an effective and reliable technique for the formation of well-defined and crystallographically aligned semiconductor nanostructures. Using $\mathrm{HgTe}-\mathrm{PbTe}$ quasi-binary semiconductor system, self-aligned $\mathrm{HgTe}$ nanocrystallites distributed three dimensionally within $\mathrm{PbTe}$ matrix were successfully formed by the simple three step heat treatment process routinely found in age hardening process of metallic alloys. Examination of the resulting nano precipitates using conventional transmission electron microscopy (CTEM) and high resolution TEM (HRTEM) reveals that the coherent $\mathrm{HgTe}$ precipitates form as thin discs along the $\{100\}$ habit planes making a crystallographic relation of $\{100\}_{\mathrm{HgTe}} / /\{100\}_{\mathrm{PbTe}}$ and $[100]_{\mathrm{HgTe}} / /[100]_{\mathrm{PbTe}}$. It is also found that the precipitate undergoes a gradual thickening and a faceting under isothermal aging up to 500 hours without any noticeable coarsening. These results, combined with the extreme dimension of the precipitates (4-5 nm in length and sub-nanometer in thickness) and the simplicity of the formation process, leads to the conclusion that controlled precipitation is an effective method for preparing desirable quantum-dot nanostructures.
\end{abstract}

Keywords : HgTe nanocrystallites, Controlled precipitation, Quantum dot nanostructure

\section{INTRODUCTION}

Semiconductor nanocrystallite is a new class of materials due to its unique properties[1,2] that make them demanding for future device applications. Especially, the quantum dot that has zero dimensionality has various technological importance because of a drastic change in the behavior of carriers. One of the important unique qualities is the quantum confinement effect, which shows an atom-like behavior due to its zero dimensionality. An extensive amount of research has been conducted over the past decade to understand fundamentals governing nanocrystallite properties as well as to develop effective fabrication methods [3,4].

The controlled precipitation process is being developed as a new way to produce a large number of quality semiconductor nanocrystals more simply than the conventional methods[5]. The concept of controlled precipitation of semiconductor nanocrystallites has much in common with that of the age hardening process routinely found in metal alloys[6]. It essentially consists of three-step heat treatments, solid solution thermal treatment, quenching and aging, conducted on an alloy system where the solubility of alloying element has considerable temperature dependence. The solid solution treatment of the alloy produces a homogeneous solid solution. By quenching to room temperature, precipitation in the supersaturated alloy is suppressed. Finally, the alloy is aged at a temperature where controlled homogeneous nucleation and growth of precipitates happens. Due to slow diffusion kinetics at the aging temperature, the resulting precipitates can be extremely small and densely populated. Controlled precipitation should be equally effective for any type of semiconductor system, either in a bulk or in a film form, in producing coherent nanocrystalline precipitates as long as the proper alloy system, composition, and heat treatment conditions are chosen.

The propose of the present study is to prove the effectiveness of controlled precipitation in the fabrication of quantum dot nanostructure focusing on the characterizations of $\mathrm{HgTe}$ nanocrystalline precipitates using conventional and high resolution transmission electron microscopy study. There are many 
parameters to be determined such as shape, size and distribution of nanocrystallites. The present study aims to provide answers to these questions and establish the revolutionary method.

\section{EXPERIMENTAL}

The alloys used in this investigation were based on PbTe containing $x$ mol. \% HgTe (PbTe- $x \mathrm{HgTe}$ ). The equilibrium $\mathrm{PbTe}$ phase has a $\mathrm{NaCl}$ structure with a lattice constant of $6.462 \AA$ and a direct bandgap of $0.31 \mathrm{eV}(300 \mathrm{~K})$. The $\mathrm{HgTe}$ phase has a sphalerlite structure with a lattice constant of $6.453 \AA$ and a bandgap of $-0.141 \mathrm{eV}(300 \mathrm{~K})$. The starting poly-crystalline stoichiometric compounds, $\mathrm{PbTe}$ and $\mathrm{HgTe}$, were synthesized by direct melting of the constituent elements (purity higher than 99.999) in sealed quartz ampoules filled with argon gas. The $\mathrm{PbTe}-x \mathrm{HgTe}$ alloys were synthesized using the same method. The resulting semiconductor alloys were each placed in a graphite crucible with a lid and heated to $970^{\circ} \mathrm{C}$ in argon for half an hour for homogenization. The ingot was then slowly cooled to $600^{\circ} \mathrm{C}$ at $1 \sim 2 \mathrm{deg} . / \mathrm{min}$. and quenched to room temperature to minimize precipitation during cooling. The ingot was heated again and held at $600^{\circ} \mathrm{C}$ for 24 hours to induce complete solid solution of $\mathrm{HgTe}$ and $\mathrm{PbTe}$. To induce precipitation, samples were aged at 300 and $400^{\circ} \mathrm{C}$ for various times, ranging from 5 to 300 hours.

Transmission electron microscopy was the primary technique used in this investigation for the characterization of the HgTe precipitate. TEM sample preparation required special attention. Our preliminary investigation indicates that the precipitation behavior changes drastically when the alloy is stored too long as a thin foil, especially when it is stored under vacuum. For this reason, TEM samples were made with minimum exposure to vacuum and heat. Properly cut ingots were bonded to copper grids, mechanically ground to form a dimple and then briefly ion milled. TEM and HRTEM characterization were conducted immediately after the sample preparation.

\section{RESULTS AND DISCUSSION}

\subsection{Observation of HgTe nucleation}

The early stage of precipitation, namely, the nucleation stage of embryos, can be detected by CTEM only when precipitates strain the matrix and produce strain fields around precipitates. Also, the detection of such strain fields means that there is a considerable lattice mismatch between precipitates and matrix. An example of such an early stage precipitation is given in figure 1, showing the precipitation of $\mathrm{HgTe}$ nanocrystallites in $\mathrm{PbTe}$ matrix, aged for 10 minutes at $300^{\circ} \mathrm{C}$. Figure $1(a)$ is obtained from a $\mathrm{PbTe}-4 \mathrm{HgTe}$ foil oriented near $<001>$ and $(b)$ near $<112>$ zone axis. Both images show the strain field images resulted from the considerable radial stress generated within the matrix. Due to the short duration time of embryo state, the exact nucleation stage is difficult to observe. However, it is believed that precipitates shown in figure 1 denote the precipitates at the nucleation stage because the nearly spherical strain field images compared to those formed at later stages.

If the total energy of spherical embryo is higher than
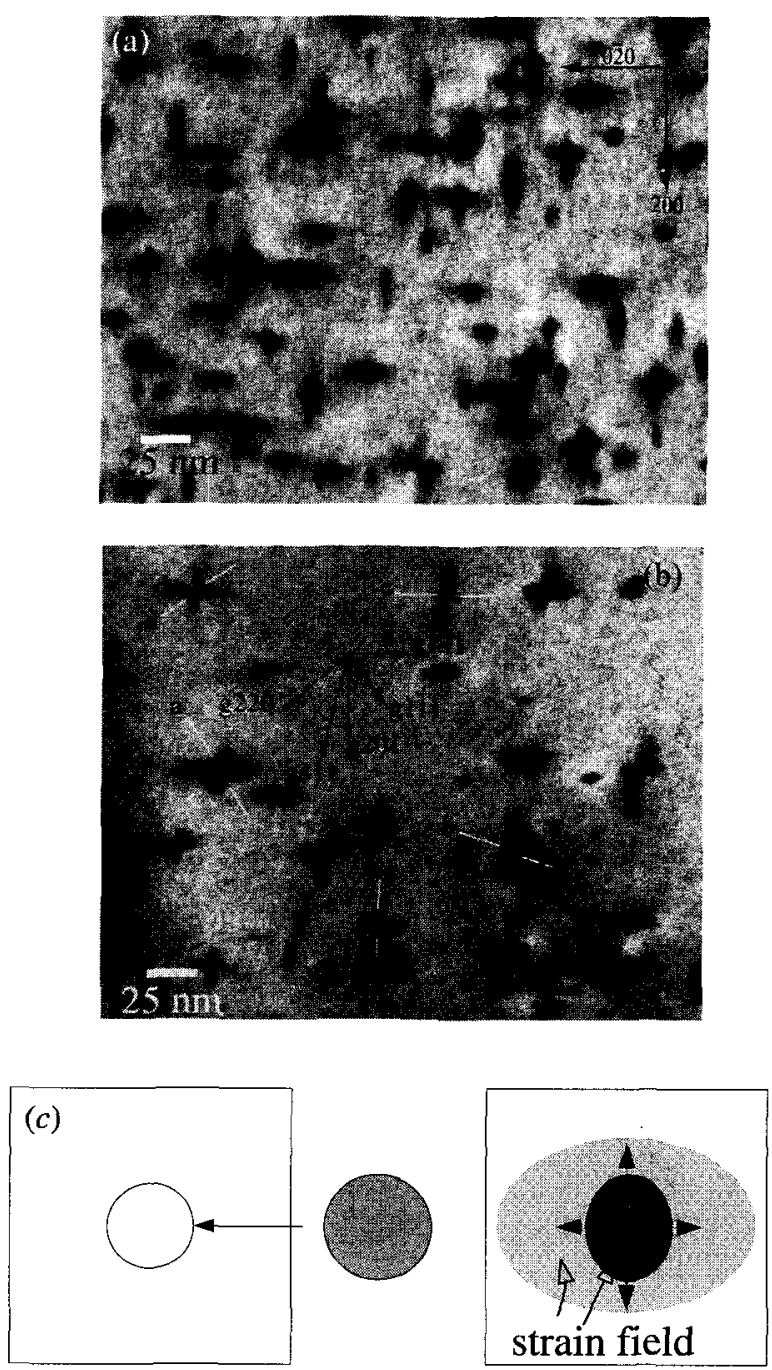

Fig. 1. TEM Bright field images of early stage precipitates of $\mathrm{PbTe}-4 \mathrm{HgTe}$ samples, which are aged for 10 minutes at $300^{\circ} \mathrm{C}$ taken at (a) 100 zone axis and (b) 112 zone axis $([\overline{1} 12])$, respectively, (c) shows a model for the generation of strain field. 
that of platelets, the spherical embryo must evolve to platelet, at the expense of interfacial energy increase, because this evolution direction is energetically favorable. In case of plate-like coherent precipitates, displacement vector could be induced normal to the precipitates[7]. Therefore, the actual growth direction of each precipitate is believed to the direction normal to each strain field direction, which will be discussed later.

Evidence of spherical shape nuclei and their subsequent growth to $\langle 001\rangle$ direction can be better analyzed from fig. $1(b)$. When the condition $g \cdot R=0(R$ being the radial displacement) is satisfied, the strain induced distortion will fail to produce contrast in the electron image, and a 'line of no contrast' normal to the operating $\mathrm{g}$ vector may be evident as suggested in fig. $1(b)$. All possible g vectors are defined in fig. $1(b)$ under [i112] multiple-beam condition. They are g220, g311, $\mathrm{g} 201, \mathrm{~g} 1 \overline{1} 1$, and $\mathrm{g} \overline{13} 1$. As shown in this micrograph, the $\mathrm{g}$ vectors, $\mathrm{g} 220, \mathrm{~g} 31 \mathrm{l}, \mathrm{g} 201, \mathrm{~g} 1 \overline{1} 1$, and $\mathrm{g} \overline{13} 1$ produce no contrast line on some precipitates $a, b, c, d$ and e, respectively. If they do not have spherical shape, at least one $\mathrm{g}$ vector can not show line of no contrast. Therefore, the strain field direction in fig. 1(b) could be interpreted at least as radial, even though not completely spherical. This analysis concludes that the precipitates in nucleation stage have become rounded and are about to evolve. Since the strain field contrast originates from the distortion of the matrix phase, the actual size of precipitates in this early stage cannot be determined. It is, however, believed to be much smaller than what is shown in fig. 1. Figure $1(c)$ shows a model explaining the strain field generated around nanocrystallites.

\subsection{Observation of HgTe nano-disc}

The mechanism of $\mathrm{HgTe}$ precipitation in $\mathrm{PbTe}-\mathrm{HgTe}$ is similar to that found in metallic systems in several respects. HgTe precipitates are found to be small and extremely well distributed, suggesting that homogeneous nucleation and growth has occurred during the precipitation stage. Similar to many cases in metallic systems, the precipitate shape is controlled by strainenergy, leading to the formation of thin disks. Fig. 2, where TEM micrographs and diffraction patterns at the $<100\rangle$ and $<110\rangle$ zone axis are shown, presents evidence of disk-shaped HgTe precipitates. For example, two sets of disks running parallel to the beam are visible in the $<100>$ axis bright field image (Fig. 2(a)). The faint streaks appearing around the main diffraction pattern also indicate the existence of disk shaped precipitates. Close examination of the bright field image and diffraction pattern also reveals that the precipitate is crystallographically aligned to the $\mathrm{PbTe}$ matrix, that is $\{100\}_{\mathrm{HgTe}} / /\{100\}_{\mathrm{PbTe}}$ and $[100]_{\mathrm{HgTe}} / /[100]_{\mathrm{PbTe}}$. Therefore, there are three sets of plates oriented in the [100], [010]
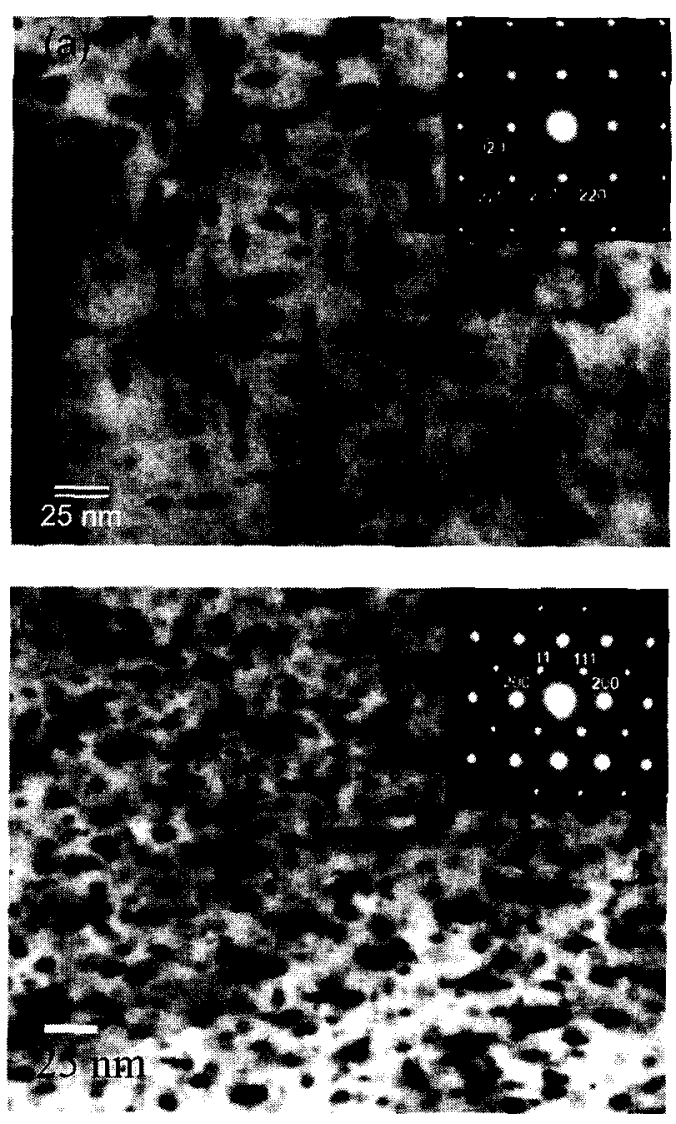

Fig. 2. TEM micrographs (bright field image and diffraction pattern) showing the formation of $\mathrm{HgTe}$ precipitates after aging 5 hours (initial stage of precipitation) at $300^{\circ} \mathrm{C}$ taken at two different zone axes: a) $<100>$; b) $<110\rangle$.

and [001] directions.

The existence of such alignment should lead to a coherent interface in which the lattice is continuous across the $\mathrm{PbTe}-\mathrm{HgTe}$ interface. When $\mathrm{HgTe}$ precipitates are examined under HRTEM, it is found that the interface is indeed coherent and is free of any apparent defects. Fig. 3 show examples of HRTEM images of early stage $\mathrm{HgTe}$ precipitates. These micrographs show (200) interference fringes taken at the $<100>$ zone axis of PbTe for a HgTe nano-disc. Note the continuity of the interference fringe. $\mathrm{HgTe}$ appears bigger in the bright field TEM micrograph (Fig. 2) than in HRTEM image (Fig. 3) due to additional contrast arising from lattice strain induced to maintain a coherent interface. Since the strain contrast obscures the true dimension of $\mathrm{HgTe}$ precipitates, the size estimate is based on the HRTEM image at the expense of statistical significance. The best estimate of disk size is determined to be $<1 \mathrm{~nm}$ thick and $4-5 \mathrm{~nm}$ in diameter. Notice also the extreme density of the $\mathrm{HgTe}$ phase, which is estimated to approach $3 \times 10^{5} / \mu^{3}$. Nanocrystals are 
nearly impossible to produce in more conventional methods with such a high density and the ability to selfalign.
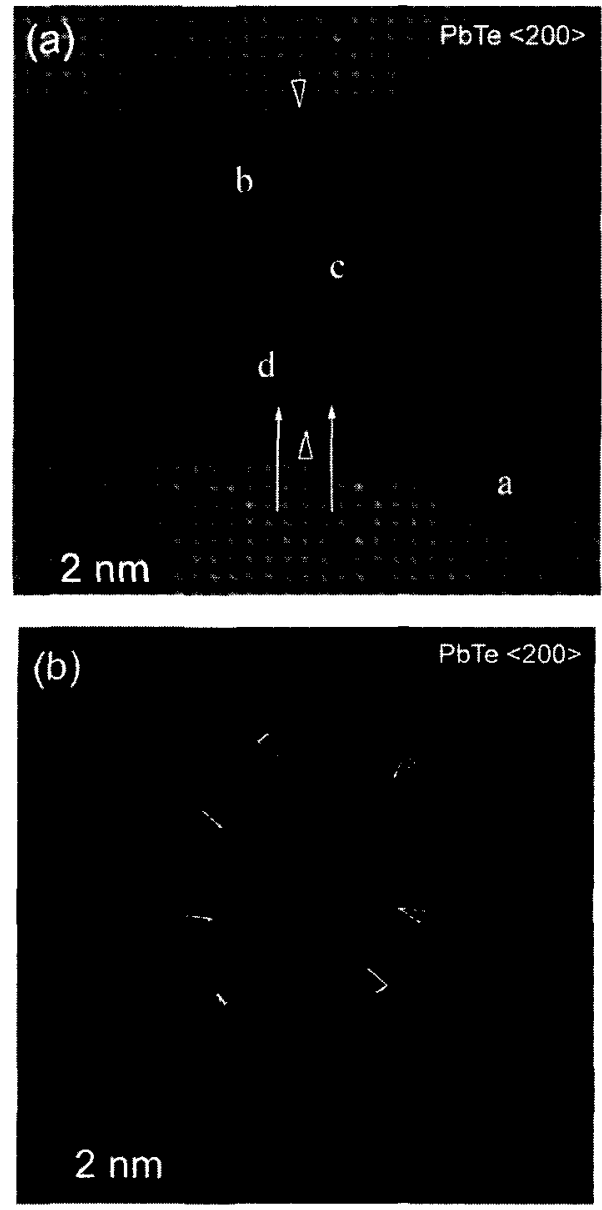

Fig. 3. Typical <001> HRTEM images of disc type precipitates in the $\mathrm{PbTe}-2.5 \mathrm{~mol}$.\% $\mathrm{HgTe}$ alloy aged for 5 hours at $300^{\circ} \mathrm{C}, \mathrm{HgTe}$ nano-disc aligned (a) parallel to beam direction, (b) perpendicular to beam direction.

\subsection{Shape evolution of HgTe precipitates}

Further observation on $\mathrm{HgTe}$ nano-discs beyond initial precipitation indicates that $\mathrm{HgTe}$ disc experiences an additional shape change. Since precipitation is completed within one hour aging, additional shape change is believed to occur during the coarsening stage. In a typical metallic alloy, the precipitates coarsen with further aging once precipitation is completed. During coarsening, the size of the precipitates increases while their number decreases. However, the HgTe precipitates do not seem to follow the normal coarsening process. Fig. 4 illustrate the evolution of precipitate image with aging time at $300^{\circ} \mathrm{C}$. It can be seen that the precipitate density is not significantly changed with aging time. The insignificant density change makes it difficult to determine the completion time of the precipitation process. However, TEM examinations of the precipitates after one hour aging reveal that the precipitation is probably completed.

While the density of precipitates appears to be constant, their shape and size evolves with aging time, but very slowly. As the aging time increases up to 300 hours, the precipitate, initially thin disk-shaped, starts to develop facets together with thickness growth. The faceted nature of the precipitate is clearly visible in the sample aged more than 300 hours both at $300^{\circ} \mathrm{C}$ and $450^{\circ} \mathrm{C}$, which is shown in figure 5. Further aging up to 500 hours shows almost the same results as that of the 300 hours aged sample. The faceting, which is the effect of interfacial energy, is evidence of the strain field reduction[8].
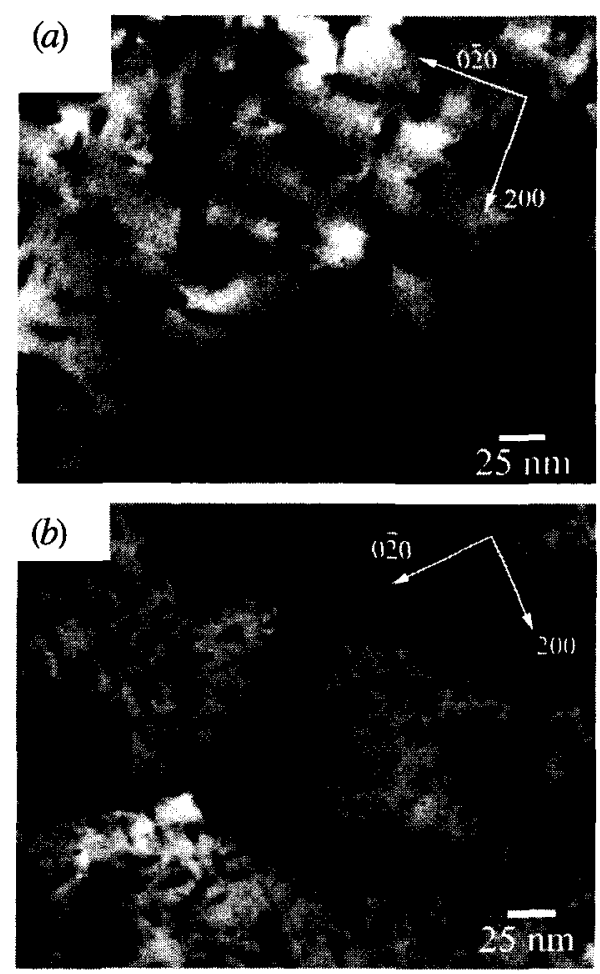

Fig. 4. TEM micrographs of PbTe-4HgTe showing the evolution of precipitation aged at $300^{\circ} \mathrm{C}$ as a function of aging time: (a) 25 hours and (b) 125 hours.

It is observed that the shape change is accompanied by the reduction in the strain field. The strain is found to decrease with the increase of aging time. Comparing the actual strain field trails in fig. 4 , the size and intensity of strain fields seem to be reduced when aging time increased from 25 hours to 125 hours. Careful examination of various strain field images obtained from various experimental conditions showed that the strain field trails are maximized in the early stage of precipitation (after 1-5 hours aging), and after that, they 
gradually decrease. Notice, also, there are still present a strain field contrast parallel to the thickness direction of precipitate and an insignificant trails on the edge of precipitate as shown in fig. 5. This means that an induced strain still remains after a prolonged aging $(300$ hours) and acts as one of the important energy acting in the morphology evolution.

Figure 6 shows $<100>$ HRTEM image of aged sample, denoting aligned $\mathrm{HgTe}$ nano dises precipitated homogeneously in $\mathrm{PbTe}$ matrix. All three types of aligned
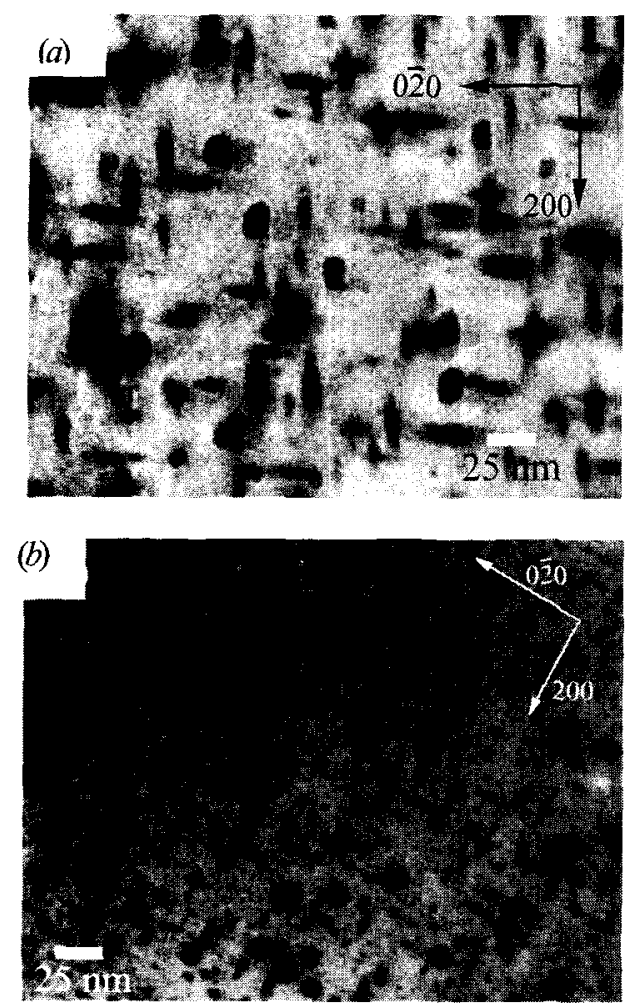

Fig. 5. TEM micrographs showing the evolution of precipitation aged at $(a) 300^{\circ} \mathrm{C}, 300$ hours and $(b) 450^{\circ} \mathrm{C}$, 300 hours.

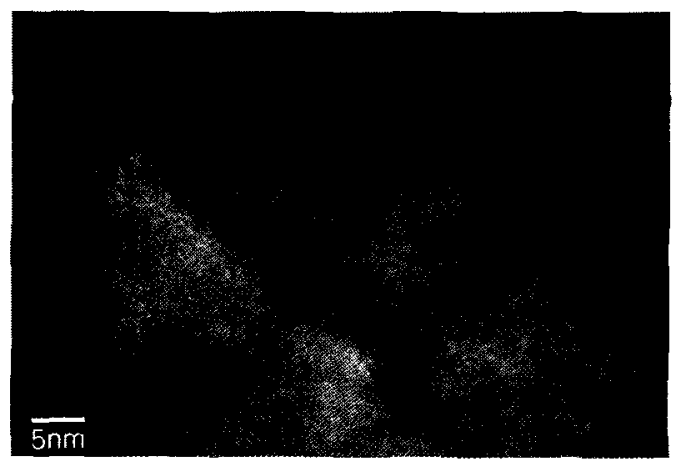

Fig. 6. The <001> HRTEM images showing the distribution of all three types of precipitates in $\mathrm{PbTe}$ $2.5 \mathrm{HgTe}$ alloy aged for 300 hours at $300^{\circ} \mathrm{C}$. nano-disc can be seen with a good periodicity.

In fig. 7 , the thickness growth of $\mathrm{HgTe}$ nano-disc is shown. As can be seen in this figure, this evolution happens by the rearrangement of atom within $\mathrm{HgTe}$ nano-discs, because their density is not changed after the thickness growth.

The practical implication of the shape change is that nanocrystals of various shapes and sizes can be made by simple heat treatment and, perhaps more importantly, with a high quality interface. It is somewhat unusual to have a precipitate system where the shape evolves without any change in interface structure. As the precipitate coarsens, an incoherent interface becomes energetically more favorable as strain energy is released at the expense of interface energy. However, the shape evolution of $\mathrm{HgTe}$ occurs without such change, suggesting the existence of a mechanism that relaxes
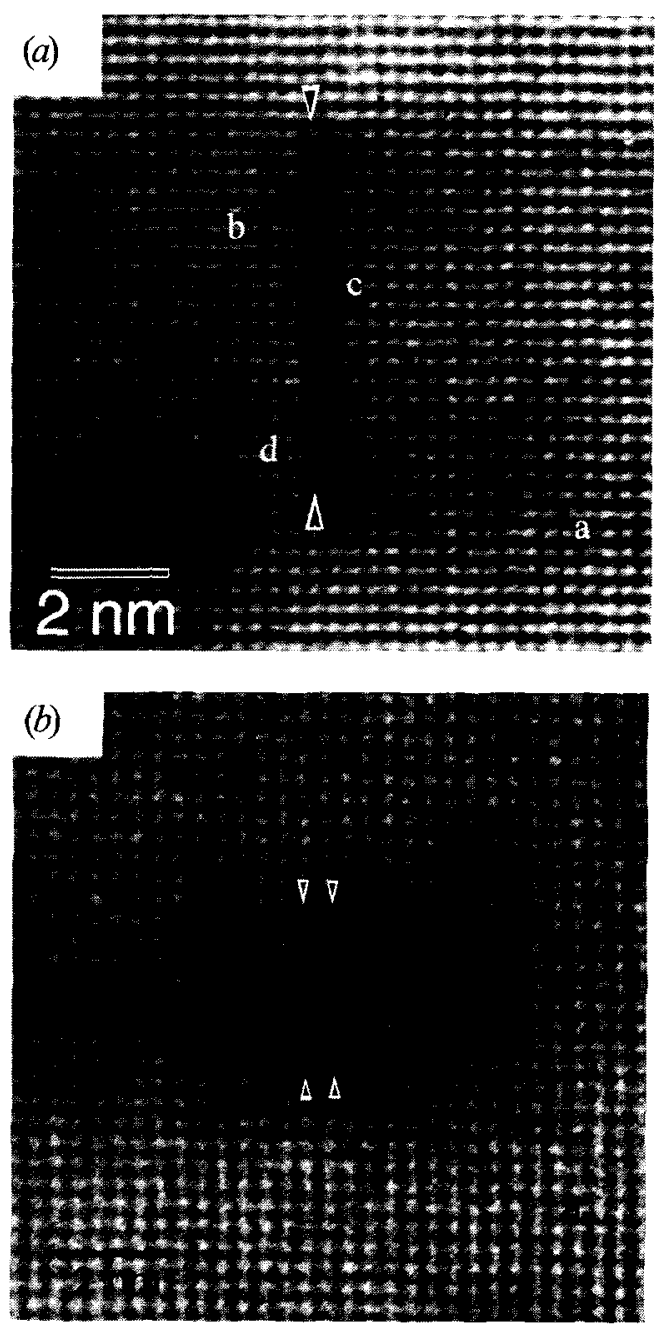

Fig. 7. HRTEM images of HgTe nano-disc showing thickness growth; aged for a) 125 hours and b) 300 hours at $300^{\circ} \mathrm{C}$. 
strain energy during coarsening. It is believed that the mechanism of strain energy relaxation is related to the disorder-order phase transformation occurring within HgTe precipitates. As shown in fig. 3 , the presence of a disk-shaped precipitate creates a long-range strain field in the PbTe matrix. The presence of such an extensive strain field is rather unexpected because the lattice mismatch between the equilibrium state of $\mathrm{PbTe}$ and HgTe is less than $0.1 \%$. This amount of strain is too small to account for the large strain field observed. HRTEM analysis indeed indicates that the PbTe matrix near the $\mathrm{HgTe}$ disk is under nearly $10 \%$ tensile strain. Both conventional TEM and HRTEM analysis show that the strain decreases with aging, which can be seen in fig. 7(a). The presence of a large tensile strain in PbTe near the HgTe disk and its subsequent reduction with the shape evolution suggest that the initial HgTe phase has a larger volume than its equilibrium state. One of the easiest ways to account for a larger equilibrium volume is with a disordered structure. Although not presented in this paper, an additional characterization [9] with Raman spectroscopy provides supporting evidences that the order-disorder phase transformation occurs within $\mathrm{HgTe}$ and is responsible for the shape evolution observed in this study. The volume contraction resulting from the ordering of HgTe nano-disc can be found by the actual measurement of HgTe lattice constant (shown as a, b, c and d) using fig. 3(a) and 7(a). The lattice constant shown as $\mathrm{c}$ in fig. 3(a) of initial $\mathrm{HgTe}$ nano-disc is decreased compared with a lattice constant shown as $\mathrm{c}$ in fig. 7(a) aged for 125 hours, which accounts for strain induced ordering and subsequent volume contraction. The detailed mechanism will be discussed in detail in a next report.

From the results discussed so far, this new method, "controlled precipitation technique" is concluded to be very effective in producing compound semiconductor nanostructure consisting high density nanocrystallites and semiconductor matrix.

\section{CONCLUSION}

Using TEM study, the following unique experimental results were obtained and analyzed for the precipitation behavior of PbTe-HgTe semiconductor alloys.

1. The controlled precipitation process in the compound semiconductor alloy systems is very effective in producing nanostructures consisting of a matrix phase of higher bandgap compound semiconductor and nanocrystals of lower bandgap semiconductor, which is ideal for quantum dot nanostructure.

2. The initial shape of precipitates is believed to be a spherical. The actual observation of a sphere, however, was very difficult due to the short duration time of such stable embryos. Only strain field contrast, generated by the spherical precipitates, can be observed using conventional TEM study.

3. Disc shaped precipitates are formed from spherical embryos as a result of high strain energy minimization involved in spherical precipitates and exist as stable precipitates nearly up to the final stage of the precipitation.

\section{REFERENCES}

[1] T. Takagahara, "Excitonic optical nonlinearity and exciton dynamics in semiconductor quantum dots," Phys. Rev. B, Vol. 36, No. 17, p. 9293, 1987.

[2] Y. Z. Hu, M. Lindberg, and S. W. Koch, "Theory of optically excited intrinsic semiconductor quantum dots," Phys. Rev. B, Vol. 42, No. 3, p. 1713, 1990.

[3] V. I. Klimov, A. A. Mikhailovsky, Su Xu, A. Malko, J. A. Hollingsworth, C. A. Leatherdale, H.-J. Eisler. and M. G. Bawendi, "Optical gain and stimulated emission in nanocrystal Quantum Dots," Science, Vol. 290, p. 314, 2000.

[4] M. Balkanski and R. F. Wallis, "Semiconductor Physics and Applications", Oxford Univ. Press, 2000.

[5] M. J. Lee and C.-U. Kim, "Investigation on selfaligned $\mathrm{HgTe}$ nano-crystals induced by controlled precipitation in $\mathrm{PbTe}-\mathrm{HgTe}$ quasi-binary compound semiconductor alloys," Physica B Vol. 304, No. 1-4. p. 267, 2001.

[6] J. W. Martin, "Precipitation Hardening", Pergamon, New York, 1968.

[7] G. Thomas and M. J. Goringe, "Transmission Electron Microscopy of Materials", Wiley \& Sons, New York, 1979.

[8] A. P. Sutton and R. W. Balluffi, "Interfaces in Crystalline Materials", Oxford University Press, New York, NY, 1995.

[9] Man-Jong Lee, "Mechanisms of precipitation and shape evolution of $\mathrm{HgTe}$ nanocrystallites in $\mathrm{HgTe}$ $\mathrm{PbTe}$ induced by controlled precipitation technique”, Ph. D. Dissertation, UTA, 2001. 